\title{
ORIGINAL
}

\section{FACTORES DE RIESGO Y MORBI-MORTALIDAD CORONARIA A LOS 28 AÑOS DE SEGUIMIENTO DE UNA COHORTE CON BAJA INCIDENCIA DE LA ENFERMEDAD: EL ESTUDIO DE MANRESA (*)}

Luis Tomás Abadal (1), Cristina Varas Lorenzo (2) Iñaki Pérez (3), Teresa Puig (2), Ignacio Balaguer Vintró (1)

(1) Servicio de Cardiología. Hospital de Sant. Pau, Barcelona

(2) Institut de Recerca: Hospital de Sant Pau, Barcelona

\section{RESUMEN}

Fundamento: Estudio de la morbimortalidad coronaria y mortalidad total asociadas a los denominados factores de riesgo coronario de una cohorte industrial de varones seguida durante 28 años.

Métodos: Población laboral de 1.059 varones de 30-59 años libres de cardiopatía en el examen inicial (1968), reexaminados cada cinco años hasta 1988 y con el examen final en 1996. Durante el tiempo de seguimiento se obtuvo información del $96,4 \%$ del total de participantes.

Resultados: Las tasas de incidencia de enfermedad coronaria, moralidar cor $10^{5}$ (personas-años de observación) fueron: 499,80; 235,80 y 925,33, respectivamente. Los nivevación) fueron: 499,$80 ; 235,80$ y 925,33 , respectivamente. Los nive-
les elevados de colesterol y el consumo habitual de cigarrillos se asociaron independientemente con la incidencia de cardiopatía coronaria y la mortalidad coronaria ajustado por edad, presión arterial, glucemia e índice de masa corporal. Los niveles elevados de colesterol sérico, glucemia y consumo de cigarrillos se asociaron independientemente con el riesgo de muerte por todas las causas.

Conclusiones: En esta población, con incidencia relativamente baja de enfermedad coronaria, el tabaco y el colesterol sérico mantuvieron el valor predictivo independiente, durante los 28 años de seguimiento.

Palabras clave: Estudio de Manresa. Incidencia. Factores de riesgo. Estudios de cohortes. Cardiopatía coronaria. cardiopatía isquémica. Mortalidad. Infarto de miocardio. Colesterol. Hipertensión. Tabaco. Indice de masa corporal.

Correspondencia:

Dr. L Tomas Abadal

Cardiología.

Hospital de St Pau.

Av. S.A.M ${ }^{\mathrm{a}}$ Claret 167

08025-Barcelona

Correo electrónico: 1tomas@hps.simtpan.es

\section{ABSTRACT}

Risk Factors and 28 Year morbimortality of Coronary Heart Disease in a Cohort with a Low Incidence of the Disease: the Manresa Study

Background: To Study the incidence of coronary heart disease (CHD) and all-cause mortality in a cohort of men followed during 28 years, and their association with serum cholesterol, systolic blood pressure, glycemia, cigarette smoking and body mass index measured at baseline.

Methods: A cohort of 1,059 men aged 30 to 59 years and free of cardiovascular diseases at baseline in 1968, was reexamined every five years until 1988. The last examination was performed in 1996 . Information was collected on $96.4 \%$ of the participants.

Results: Incidence and mortality rates from CHD and from allcauses of death per $10^{5}$ person-years of observation were 499.80 235.80 y 925.33 , respectively. At the end of follow-up, high levels of serum cholesterol and smoking were independently associated with the incidence and mortality from CHD controlling by age, blood pressure, glycemia and BMI. Serum cholesterol, hyperglycemia and smoking were independently associated with all-cause mortality.

Conclusions: In this industrial cohort of men, with relative low incidence of CHD, smoking and serum cholesterolat baseline remained associated with the incidence of CHD through 28 years of observation.

Key words: Manresa study. Risk factors. Cohort studies. Incidence. Ischaemic heart disease. Coronary heart disease. Mortality. Myocardial infarction. Cholesterol. Hypertension. Tobacco. body mass index.

(*) El examen del 28 aniversario se ha realizado con ayudas de ACARD y ALMIRALLPRODESFARMA, la colaboración de la FUNDACION DR ECHEVARNE y la cooperación del personal del CENTRE HOSPITALARI-UNITAT CORONÀRIA y de los otros hospitales de la ciudad de Manresa. 


\section{INTRODUCCIÓN}

En España, las enfermedades cardiovasculares (ECV) constituyen la primera causa de mortalidad ${ }^{1}$.

En el área mediterránea, las tasas de incidencia y mortalidad por cardiopatía coronaria $(\mathrm{CC})$ son relativamente bajas comparadas con las observadas en EE UU o en el norte de Europa ${ }^{2,3}$. Los primeros estudios prospectivos poblacionales para la identificación del riesgo coronario se iniciaron en 1948 en EE UU. Ancel Keys estudió una cohorte de hombres de negocios y profesionales de Minneapolis ${ }^{4}$ y Thomas Dawber estudió una muestra de la población de la ciudad de Framingham ${ }^{5}$. Desde entonces, en numerosos estudios prospectivos realizados en países industrializados ${ }^{6-9}$ se ha demostrado la estrecha relación entre nivel de colesterol, presión arterial y consumo de cigarrillos con el riesgo de padecer un episodio coronario agudo. Por otro lado, hay muy poca información acerca del valor predictivo de los factores de riesgo en poblaciones con tasas bajas de enfermedad coronaria ${ }^{10,11}$.

En 1968 iniciamos un estudio prospectivo de una cohorte de 1.059 varones de 30 a 59 años, sanos, trabajadores en una factoría de la ciudad de Manresa ${ }^{12}$. Esta cohorte fue controlada durante 28 años, con exámenes de seguimiento cada cinco. El estudio finalizó en $1996^{13,14}$.

El estudio de Manresa se inició en una época en que los estudios prospectivos longitudinales de población se introdujeron en Europa, siguiendo la iniciativa americana. En el «estudio siete países» ${ }^{7}$, comparando los datos americanos con los europeos, se comprobó que no existen poblaciones con niveles bajos de colesterol y tasas altas de enfermedad. Esto ponía de manifiesto la importancia del tipo de alimentación y hábitos de vida para explicar las diferencias en la frecuencia de este tipo de enfermedades en poblaciones distintas.
En este trabajo describimos la asociación de los factores de riesgo coronario medidos en el examen inicial con las tasas de morbimortalidad coronaria aparecidas en 28 años de seguimiento.

\section{POBLACIÓN, MATERIAL Y METODO}

\section{Población en estudio}

La ciudad de Manresa en 1968 tenía 58.108 habitantes. El 60\% habían nacido en Cataluña. El 17\% restante llevaban más de quince años viviendo aquí. Todos los hombres de 30 a 59 años empleados y trabajadores de la factoría Pirelli, ubicada en Manresa, fueron seleccionados. De ellos, 1.059, sanos, sin cardiopatía, constituyeron la cohorte del estudio.

\section{Primer examen}

En el examen inicial administramos un cuestionario estandarizado de consumo de cigarrillos y registro de antecedentes personales y familiares de enfermedad cardiovascular (ECV), diabetes, accidente vascular cerebral (AVC), hipertensión arterial, hipercolesterolemia y muerte súbita. Medimos el peso corporal y la altura, así, obtuvimos el índice de masa corporal (IMC). La presión arterial, se midió con un esfigmomanómetro de mercurio, en el brazo derecho, con el participante en reposo durante cinco minutos. Las presiones sistólica y diastólica fueron definidas en la primera y quinta fase de los ruidos de Korotkoff, respectivamente.

El electrocardiograma basal (ECG) de 12 derivaciones se interpretó con el código de Minnesota. Dos codificadores entrenados leyeron y clasificaron los trazados por separado. Los desacuerdos fueron revisados por un tercer lector de referencia.

Se obtuvo una muestra de sangre por venopunción después de 12 horas de ayuno com- 
pleto. Se determinaron los niveles plasmáticos de colesterol y glucosa con las técnicas de Sols y Folin-Wu respectivamente. El control de calidad del laboratorio fue doble: interno del propio laboratorio, y externo del laboratorio de referencia de la OMS en Praga.

\section{Seguimiento y registro de nuevos casos}

Hemos obtenido información completa de 1.021 de los 1.059 participantes iniciales. La supervivencia y el periodo libre de eventos coronarios se definió para cada individuo desde el día de su inclusión hasta la aparición de la primera crisis coronaria, muerte, pérdida de información, o estar vivo el 30 de diciembre de 1996.

Los exámenes de seguimiento se realizaron en 1973, 1978, 1983 y 1988. En 1996 se realizó el examen final. La participación de los supervivientes en estos exámenes ha sido alta $(92 \%)$. En cada examen se administró un cuestionario estandarizado para obtener la información del estado de salud y de los episodios que provocaron una baja laboral superior a tres semanas. Obtuvimos información para la validación de los nuevos casos a partir de los informes del hospital o del médico que habían atendido al participante. Desde 1972 dispusimos de una unidad coronaria (UC) ubicada en la ciudad, a la que acudieron casi todos los pacientes con un evento coronario agudo. Durante el seguimiento hemos tenido acceso a las historias clínicas de los ingresados en la UC o en otros centros sanitarios de la ciudad. La información de las muertes se obtuvo a través de los certificados de defunción y de los comunicados de muerte, extendidos por la compañía de seguros. También tuvimos información proporcionada directamente por los familiares. Los participantes jubilados eran controlados por los médicos de la seguridad social.

La información de las muertes y de los nuevos casos de CC fue revisada indepen- dientemente por dos miembros del equipo y clasificados según los criterios diagnósticos establecidos. En caso de discrepancia intervino un tercer evaluador. Las causas específicas de muerte se clasificaron según la novena revisión del código internacional.

La morbi-mortalidad se definió con los criterios diagnósticos estandarizados de la OMS, similares a los utilizados en otros estudios internacionales coincidentes en el tiempo.

Mortalidad Total: Muerte por todas las causas

Cardiopatía coronaria fatal: Casos fatales de infarto de miocardio o muerte súbita, presumiblemente debida a enfermedad coronaria (códigos ICD9 410-413, 795, 427.2 y 427.9). La muerte súbita se definió como la ocurrida en el intervalo de una hora desde el inicio de los síntomas, o acaecida, sin testigos, en personas sin diagnostico previo de cardiopatía coronaria ni otras enfermedades presumiblemente mortales.

Incidencia de cardiopatía coronaria: Nuevos casos de infarto de miocardio, fatal o no fatal. Para el diagnóstico de infarto de miocardio, se exigieron criterios clínicos, enzimáticos y electrocardiográficos según los criterios del registro de infarto de miocardio de la OMS.

\section{Análisis estadístico}

Las tasas de incidencia se calcularon por persona- años de observación (p.a.o.). Ajustamos por edad con el método directo de estandarización. La referencia fue la distribución de edad de toda la cohorte. La asociación entre factores de riesgo en el examen inicial con morbi-mortalidad se hizo con el análisis de regresión de riesgos proporcionales de Cox. El análisis multivariado se basó en 1.041 observaciones con información completa. 
La asociación entre los factores y el riesgo coronario o muerte durante el tiempo de observación fue calculada según categorías preestablecidas. La presión arterial fue clasificada en tres niveles según cifras de sistólica y diastólica: deseable $(\leq 129 \mathrm{~mm} \mathrm{Hg} / \leq 84$ $\mathrm{mm} \mathrm{Hg}$.), presión arterial limítrofe (130139/ 85-89 mm Hg.) e hipertensión establecida $(\geq=140 / \geq 90 \mathrm{~mm} \mathrm{Hg})$.

El colesterol plasmático se clasificó en cuatro grupos: deseable ( $\leq 199 \mathrm{mg} / \mathrm{dl})$, limítrofe (200-239 mg/dl), hipercolesterolemia moderada (240-299 mg/dl) y severa $(\geq 300$ $\mathrm{mg} / \mathrm{dl})$.

Se consideraron dos grupos en base a los valores de glicemia en ayunas: inferiores a $110 \mathrm{mgr} / \mathrm{dl}$ y superiores a esta cifra, o con diabetes conocida.

Los participantes fueron clasificados en fumadores y no fumadores, y en tres subgrupos según el índice de masa corporal (IMC): < $23 \mathrm{Kg} / \mathrm{m}^{2}$, entre 23 y $27 \mathrm{Kg} / \mathrm{m}^{2}$, y > 27 $\mathrm{Kg} / \mathrm{m}^{2}$

\section{RESULTADOS}

\section{Factores de riesgo coronario al inicio}

En la tabla 1 presentamos la prevalencia de los factores de riesgo en el examen inicial, por decenios. La edad media fue: 40,9 años.

El $66 \%$ del total tenían presión arterial deseable y el 18,6\% limítrofe. Con hipertensión establecida se halló al 15,4\%. La prevalencia de hipertensión arterial aumentaba con la edad: de un 6,7\% en menos de 39 años a un $36 \%$ entre 50 y 59 años.

Niveles de colesterol plasmático deseable (<=199 mg/dl) se observó en el 28,1\%. Nivel limítrofe en el 34,4\%. Hipercolesterolemia moderada (240-299 mg/dl) en el 33,7\%, y severa $(>=300 \mathrm{mg} / \mathrm{dl})$ en el 3,8\%. La propor- ción de individuos con hipercolesterolemia severa, aumentaba a partir de los 40 años.

Asimismo, la prevalencia de hiperglucemia aumentaba con la edad. El 4,9\% del total tenían una cifra de glucemia basal por encima de 126 mg/dl o se sabían diabéticos. La proporción aumenta desde 2,9\% para el grupo de 30-39 años al 10,3\% para los de 50-59 años. El 4,8\% tenían cifras de glucemia consideradas como prediabetes (110-125 $\mathrm{mg} / \mathrm{dl})$.

Eran fumadores habituales el $67 \%$, y de ellos el $34 \%$ eran grandes fumadores. El $59,1 \%$ tenía un IMC entre $23-27 \mathrm{Kg} / \mathrm{m}^{2}$, el $22,6 \%$ tenía sobrepeso, y, el 18,3\% tenían un IMC inferior a $23 \mathrm{Kg} / \mathrm{m}^{2}$.

\section{Incidencia de cardiopatía coronaria, muerte coronaria y muerte por todas las causas}

En el periodo de 28 años de seguimiento murieron 259 participantes. (tabla 2). En 66, la muerte se atribuyó a enfermedad coronaria. La tasa de muerte por todas las causas fue 925,33 por 105 persona-año de observación. La tasa de mortalidad coronaria fue de 235,80. En 28 años, 135 individuos presentaron un primer episodio coronario agudo. La tasa de incidencia de cardiopatía coronaria fue 499,80 por 105 p.a.o. Tanto las tasas de incidencia como de mortalidad aumentaron con la edad.

\section{Morbimortalidad coronaria y mortalidad total por categorías de los factores de ries- go al inicio}

\section{Riesgo absoluto}

En general, se observó una relación positiva y creciente entre el nivel de los principales factores de riesgo coronario en el examen inicial y la aparición de nuevos casos de cardiopatía coronaria (tabla 3), 


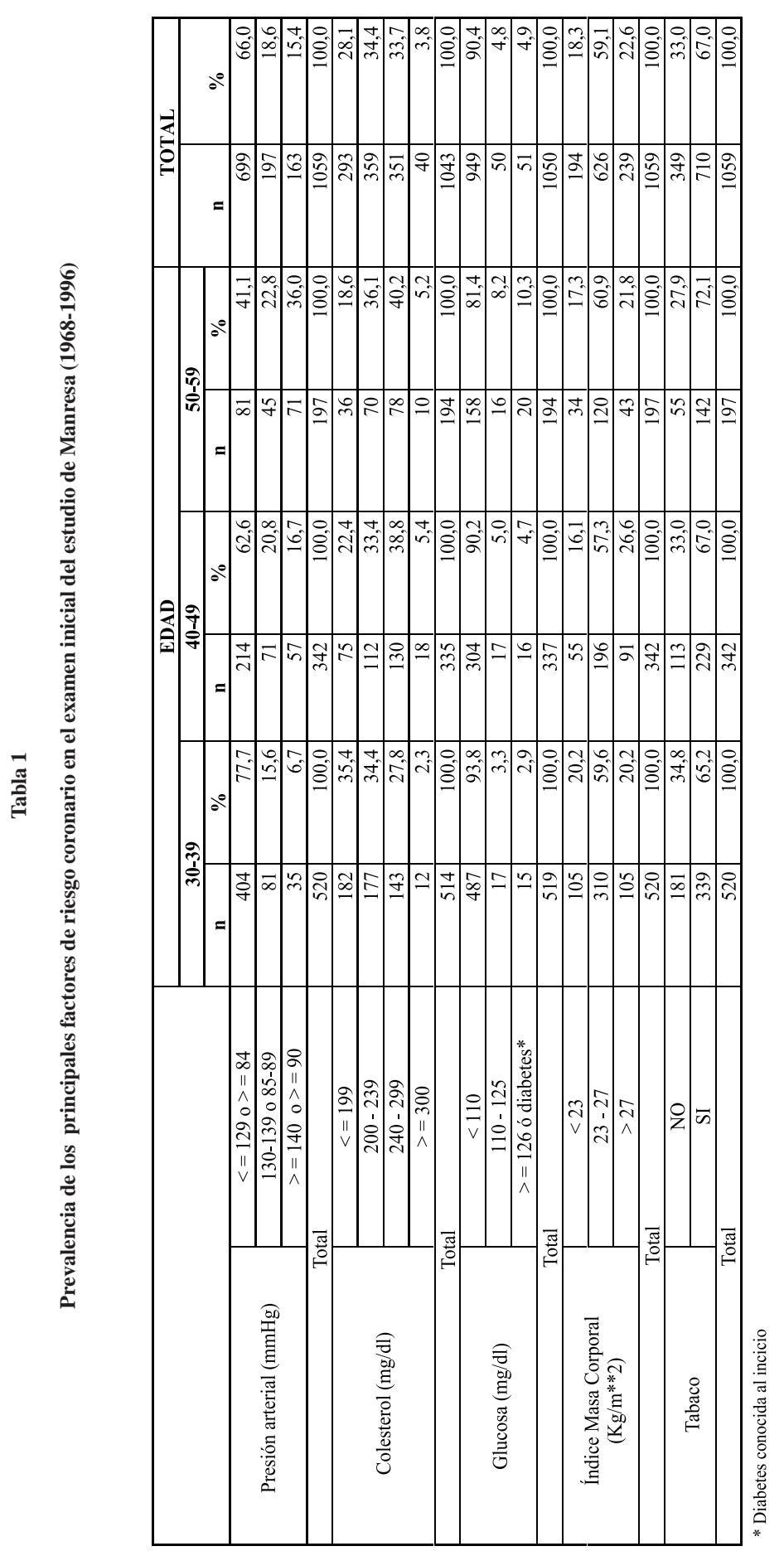

Rev Esp Salud Pública 2004, Vol. 78, N. ${ }^{\circ} 2$ 


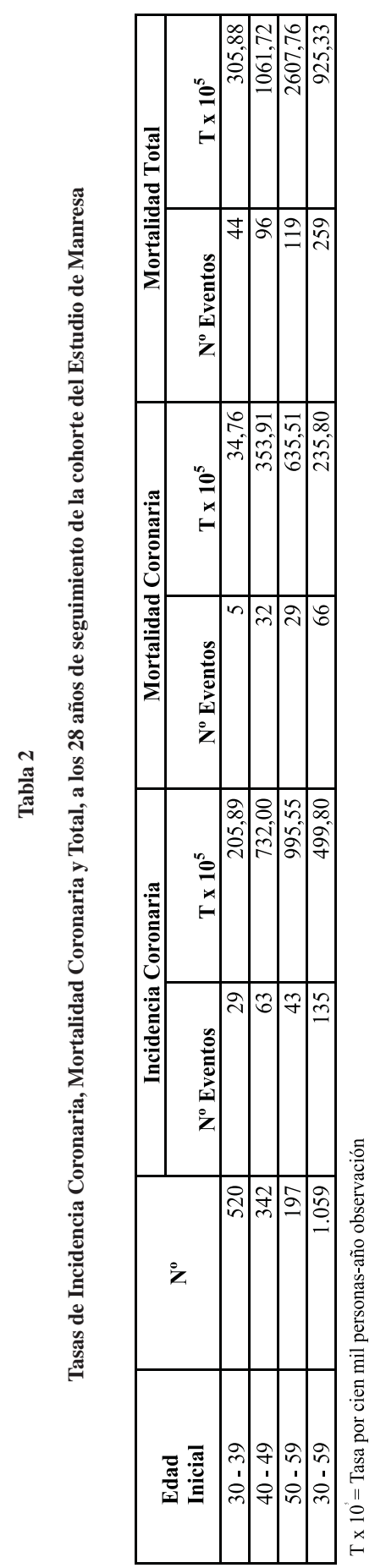


Tabla 3

Tasas de incidencia coronaria, mortalidad coronaria y total, ajustadas por la edad, por categorías predeterminadas de los principales factores de riesgo coronario en el examen inicial del estudio de Manresa (1968-1996)

\begin{tabular}{|c|c|c|c|}
\hline & \multicolumn{2}{|c|}{ Tasas por 10 personas año de observación } \\
\hline & $\begin{array}{c}\text { Incidencia } \\
\text { coronaria }\end{array}$ & $\begin{array}{c}\text { Mortalidad } \\
\text { Total }\end{array}$ \\
\hline Presión arterial sistólica & 493,94 & 232,9 & 913,5 \\
$<=129$ o $=84$ & 587,6 & 275,93 & 848,17 \\
$130-139$ o $85-89$ & 519,55 & 237,21 & 1177,88 \\
$>=140$ o $=90$ & & & 933,98 \\
\hline Glucosa & 501,88 & 228,24 & 1485,82 \\
\hline 110 & 691,17 & 438,96 & 1038,76 \\
\hline$=110$ o diabéticos* & 322,94 & 130,21 & 824,01 \\
\hline Colesterol & 442,31 & 198,59 & 143,34 \\
$200-239$ & 704,21 & 338,71 & 1216,43 \\
$240-299$ & 846,77 & 468,87 & 900,31 \\
$>=300$ & & & 1014,75 \\
\hline Indice Masa Corporal & 590,93 & 346,49 & 740,02 \\
\hline 23 & 519,32 & 234,34 & 1090,69 \\
\hline Tabaco & 496,64 & 227,59 & \\
\hline
\end{tabular}

excepto para la presión arterial y el IMC. Tanto la mortalidad como la incidencia de cardiopatía coronaria fue algo superior en los niveles intermedios de presión arterial sistólica y diastólica. Las tasas de mortalidad total y de morbimortalidad coronaria fueron más elevadas en los individuos con un IMC inferior a $23 \mathrm{Kg} / \mathrm{m}^{2}$ en comparación con las observadas en los otros grupos con IMC superior. La curva de la relación entre tasas de mortalidad total y niveles de colesterol tenía forma de «U». La tasa de mortalidad total era más alta en niveles altos de presión arterial y en los diabéticos conocidos o con niveles de glucemia superiores a $110 \mathrm{mg} / \mathrm{dl}$.

La relación entre colesterol y enfermedad coronaria fue positiva y progresiva. Lo mismo ocurrió con el tabaquismo, en el que además se puede observar un gran aumento de la mortalidad por todas las causas en el grupo de fumadores.

\section{Riesgo relativo}

La tabla 4 presenta los riesgos relativos de incidencia coronaria, mortalidad coronaria y mortalidad total ajustados por edad y el resto de factores, respectivamente. Los niveles de colesterol superiores a $240 \mathrm{mg} / \mathrm{dl}$ y el consumo de cigarrillos se asociaron independientemente con el riesgo de primer evento coronario. El riesgo de eventos fatales fue de casi 4 veces superior con cifras de colesterol =>300 $\mathrm{mg} / \mathrm{dl}$, respecto a los niveles considerados como deseables. En los fumadores, el riesgo fue doble, a todos los niveles de colesterol.

El tabaquismo, la hipercolesterolemia severa, la hiperglicemia y la diabetes se asociaron independientemente con la mortalidad total.

En la tabla 5 presentamos el riesgo relativo de incidencia de cardiopatía coronaria 


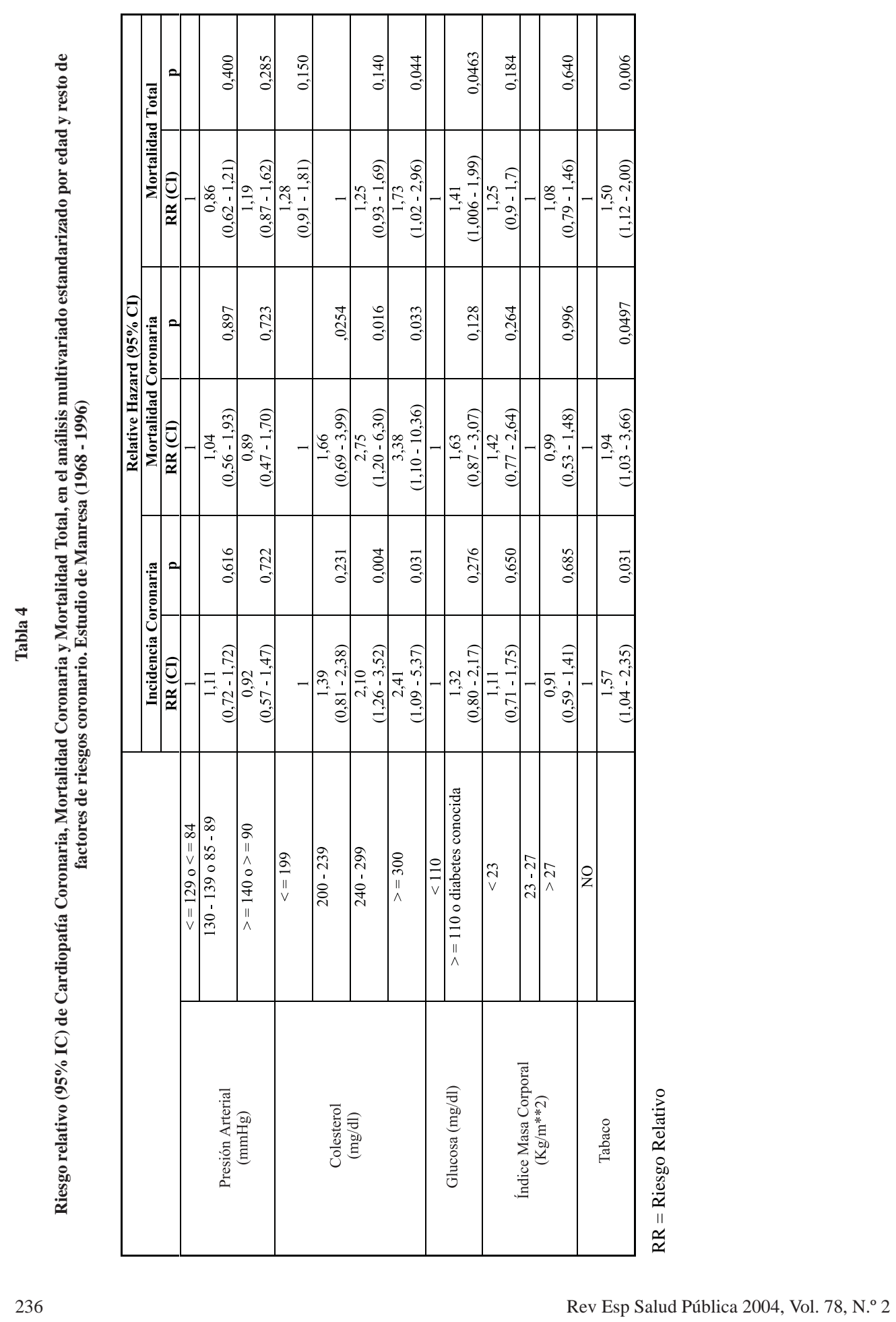


FACTORES DE RIESGO Y MORBI-MORTALIDAD CORONARIA A LOS 28 AÑOS..

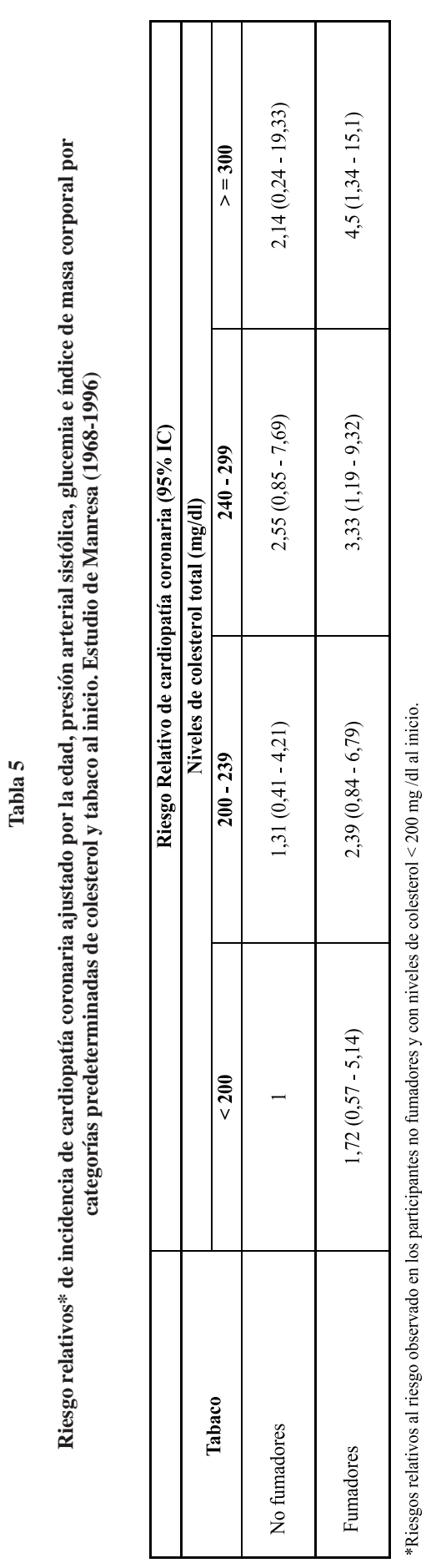

Rev Esp Salud Pública 2004, Vol. 78, N. ${ }^{\circ} 2$ 
según los niveles iniciales del colesterol y consumo de tabaco, ajustados por edad, presión arterial, glicemia e IMC. Los fumadores con hipercolesterolemia severa presentaron un riesgo casi 5 veces superior de padecer un primer evento coronario con respecto a los no fumadores con niveles de colesterol deseable. El consumo de tabaco duplicó el riesgo de cardiopatía coronaria, a cualquier nivel de colesterol considerado.

\section{DISCUSION}

El estudio de Manresa es el primer estudio prospectivo de cardiopatía coronaria en España en una muestra de población de varones sanos, trabajadores en una empresa de la industria del caucho. Pretendemos considerar esta muestra como representativa de la población general. No obstante, el fácil seguimiento de esta cohorte, constituyó una oportunidad y una ventaja para conseguir datos epidemiológicos sobre enfermedades cardiovasculares en España, por otro lado inexistente hasta entonces, sin limitar la validez interna de los resultados. obtuvimos información completa de la mayoría de los participantes durante el seguimiento. Los exámenes periódicos, las historias y los informes médicos, los certificados de defunción y los contactos directos con médicos y personal sanitario auxiliar que atendían a este grupo de población han sido básicos para conseguirlo.

En el examen inicial se encontraron niveles de colesterol relativamente altos, baja prevalencia de hipertensión arterial y alto porcentaje de fumadores. En comparación con otras cohortes, el nivel de colesterol era similar al de Bélgica, el más alto del estudio de colaboración de la OMS realizado también en población laboral. En este estudio, participaron poblaciones del Reino Unido, Bélgica, Italia, Polonia y España. El nivel de colesterol en la cohorte de Manresa es similar al de las poblaciones del sur de Europa del estudio ERICA ${ }^{15,16}$. Las cohortes medi- terráneas del estudio de Siete Países, tenían niveles de colesterol más bajos una década antes ${ }^{7}$. El nivel relativamente alto del colesterol hallado en nuestro estudio puede explicarse, en parte, por diferencias metodológicas, pero también, por los hábitos alimenticios de nuestra población, similares a los de los países industrializados del centro y norte de Europa.

La frecuencia de hipertensión arterial en el examen inicial posiblemente se ha subestimado debido a la inclusión de individuos relativamente sanos, igual que en otras cohortes industriales. En estudios posteriores realizados en Cataluña se hallaron tasas similares de prevalencia de hipertensión.

En el estudio europeo de la OMS la muestra de población catalana tenía las tasas más bajas $^{21}$. Asimismo, se hallaron tasas comunitarias relativamente bajas de hipertensión arterial en el centro Catalonia del Proyecto MONICA $^{17}$.

La proporción de fumadores fue alta y similar a la de otros estudios ${ }^{21-23}$. Desde 1957 hasta 1980 hubo un aumento constante y sostenido del consumo de tabaco en Espa$\tilde{\text { na }}{ }^{18}$.

Nuestra tasa de mortalidad cardiovascular es similar a la de otros estudios de población del área del Mediterráneo ${ }^{1,7,22}$. Para facilitar la comparación utilizamos los mismos criterios de estandarización de diagnósticos de muerte e incidencia de enfermedad coronaria del estudio «siete países» y del estudio multicéntrico europeo de factorías de la OMS. En el estudio «siete países», el diagnóstico de nuevo caso de cardiopatía coronaria se basó en la información obtenida por los equipos locales. Este procedimiento subestima la incidencia de cardiopatía coronaria, en especial el diagnóstico de infarto de miocardio definido ${ }^{7}$. Las diferencias en la detección de los casos de infarto de miocardio no fatal pueden afectar la comparación de la morbilidad. En el estudio de Manresa la 
información de los nuevos casos de infarto de miocardio se facilitó por la existencia desde 1972 de una unidad coronaria en la ciudad.

Los datos de 15 años de seguimiento del estudio «siete países» mostraban diferencias importantes de las tasas de mortalidad coronaria entre las cohortes estudiadas ${ }^{25}$. En los 15 primeros años de observación las tasas de mortalidad estandarizadas por edad en individuos de 40-59 años de Manresa eran muy similares a las de la cohorte italiana de los ferroviarios de Roma ${ }^{14}$. Sin embargo, las tasas de mortalidad observadas en Manresa a los 28 años de seguimiento son más bajas de las esperadas según el nivel de colesterol y consumo de tabaco del examen inicial. Para explicar estos hechos puede argumentarse que el tiempo de exposición a los factores de riesgo no ha sido suficiente para el desarrollo de la enfermedad, o bien que en nuestra población hay factores protectores genéticos o ambientales. Se ha atribuido este papel a la dieta, por el alto contenido de vitaminas $\mathrm{E}$ y $\mathrm{C}$, además de otros elementos antioxidantes. Las poblaciones del sur de Europa tienen niveles plasmáticos altos de estas vitaminas, como demostró un subgrupo del estudio MONICA ${ }^{20}$. A este fenómeno los franceses lo han definido como «la paradoja francesa» ${ }^{21}$, aunque en este caso deben considerarse otros factores capaces de neutralizar a esta «aparente paradoja» 22 .

A pesar de que nuestro estudio incluye un número limitado de factores de riesgo, los resultados son coherentes con los de otros estudios prospectivos ${ }^{4-9}$. Nuestros datos confirman el valor predictivo de riesgo coronario del colesterol en poblaciones, por otro lado, con tasas bajas de la enfermedad, tal y como ya reportamos en la publicación a los 20 años de seguimiento del estudio de Man$\operatorname{resa}^{10,14}$.

Las diferencias en las covariables incluidas en el análisis multivariado y las características propias de cada población son ele- mentos importantes al establecer comparaciones. La edad, el nivel de colesterol y el consumo de cigarrillos han sido los factores más importantes en la predicción del riesgo coronario después de 28 años en el estudio de Manresa, igual que en otras cohortes de varones que han sido seguidas durante un largo periodo de tiempo ${ }^{23}$. No obstante, la presión arterial no ha tenido el mismo valor predictivo al extenderse el tiempo de seguimiento, debido en parte a la edad relativamente joven de esta población cuando fue incluida en el estudio. El papel de la presión arterial como marcador de riesgo coronario fue superior en poblaciones de edad inicial más avanzada, como en el estudio «siete países» ${ }^{29}$. Hay que tener en cuenta además que en el presente análisis no se han considerado los cambios individuales en los factores de riesgo ocurridos durante el seguimiento, asumiéndose que la clasificación de los participantes según los niveles de los factores de riesgo persiste a lo largo del tiempo. Con el envejecimiento, el desarrollo de hipertensión y la aparición de diabetes debe haber sido importante. Son pues necesarios análisis adicionales para establecer cuál es la contribución independiente de cada factor a lo largo del tiempo en el riesgo de cardiopatía coronaria. No obstante, la cohorte filandesa de siete países mostró que el riesgo relativo de muerte coronaria asociado con el colesterol sérico y la presión arterial no cambió en el transcurso de 25 años. Por el contrario, en los fumadores de menos de 10 cigarrillos/día el riesgo relativo disminuía con el tiempo, debido al importante porcentaje de individuos que dejaban de fumar durante el tiempo de seguimiento. No tener en cuenta este hecho en el análisis lleva a la errónea conclusión de que los fumadores moderados no tenían aumentado el riesgo de muerte coronaria $^{29}$.

Con un seguimiento más largo, la hiperglicemia inicial y la diabetes mantienen su papel predictivo de mortalidad total y posiblemente de morbi-mortalidad coronaria. Estudios prospectivos previos habían demostrado la 
contribución de la diabetes, hiperglucemia, resistencia insulínica, hiperinsulinemia e intolerancia a la glucosa en la aparición de enfermedades cardiovasculares ${ }^{24-26}$. La diabetes se asocia a hipertensión arterial e hipercolesterolemia ${ }^{27}$. En sujetos diabéticos se ha demostrado un aumento significativo de la coaguabilidad sanguínea, agregabilidad plaquetaria, descenso de la actividad fibrinolítica y disfunción endotelial ${ }^{28}$.

Los resultados obtenidos durante 28 años de observación de esta cohorte son consistentes con los de otros estudios prospectivos. Se ha observado la asociación fuerte e independiente del consumo de cigarrillos y de la hipercolesterolemia con la morbi-mortalidad coronaria ${ }^{29,30}$. Podemos afirmar que las tasas de incidencia y mortalidad coronaria en nuestro estudio son relativamente bajas. En estudios prospectivos previos de poblaciones del área del Mediterráneo se hallaron tasas muy similares. En todos ellos, igual que en el estudio de Manresa, el papel predictivo de los principales factores de riesgo coronario es claro y contundente.

Creemos que la información obtenida es útil en la predicción del riesgo cardiovascular en nuestra población, y, por tanto, de gran valor en la planificación de campañas de prevención cardiovascular. Desearíamos poder contar con otros estudios prospectivos sobre enfermedades cardiovasculares en población general en España, para poder validar nuestros datos.

\section{AGRADECIMIENTOS}

A la asistencia y colaboración en el trabajo de campo de Gloria Durán Ruiz, del personal del servicio médico de empresa de Pirelli SA de Manresa y el soporte administrativo de Rufina Marco.

\section{BIBLIOGRAFIA}

1. Villar Álvarez F, Banegas Banegas JR, Rodríguez Artalejo F, del Rey Calero J. Mortalidad cardiovas- cular en España y sus comunidades autónomas (1975-1992). Med Clin (Barc) 1998; 110: 321-7.

2. Tunstall-Pedoe H Kuulasma K, Mähönen M, Tolonen H, Ruokokosky E, Amouyel Ph for the WHOMONICA Project. Contribution of trends in survival and coronary heart disease mortality: 10-year results from 37 WHO-MONICA Project populationa. Lancet 1999; 353: 1547-57.

3. Sans S, Kesteloot H, Kromhout D on behalf of the Task Force, The burden of cardiovascular disease mortality in Europe. Task Force of the European Society of Cardiology on Cardiovascular Mortality and Morbidity Statistics. Eur Heart J 1997; 18: 1231-48.

4. Keys A, Taylor HL, Blackburn H, Brozek J, Anderson JJ, Simonson E. Coronary heart disease among Minnesota business and professional men followed fifteen years. Circulation 1963; 28: 381-95.

5. Dawber TR, The Framingham Study. The epidemiological atherosclerotic disease. Cambridge MA and London UK: Harvard University Press; 1980.

6. The Pooling Research Group Relationship of blood pressure, serum cholesterol, smoking habit, relative weight and ECG abnormalities to incidence of major coronary events: final report of the Pooling Project. J Chron Dis 1978; 31: 201-71.

7. Keys A, Aravanis C, Blackburn H et al. Seven Countries: A multivariate analysis of death and coronary heart disease. Cambridge, MA and London, UK: Harvard University Press; 1980.

8. Regland DR, Brand RJ. Coronary Heart Disease Mortality in the Western Collaborative Group Study: Follow-up experience of 22 years. Am J Epidemiol 1988; 127: 462-75.

9. Neaton JD, Wentworth D, for the Multiple Risk Factor Intervention Trial Research Group. Serum cholesterol, blood pressure, cigarette smoking and death from coronary heart disease. Overall findings and differences by age for 316,099 white men. Arch Intern Med 1992;152: 56-64.

10. Fraser GE, Straham TM, Sabate J, Beeson WL, Kissinger D. Effects of traditional coronary risk factors on rates of incident coronary events in a low-risk population. The Adventist Health Study. Circulation 1992; 86:406-13.

11. Chen Z, Peto R, Collin R, MacMahon S, La J, Li W. Serum cholesterol concentration and coronary heart disease in populations with low cholesterol concentrations. BMJ 1991 303: 276-82. 
12. Tomás Abadal L, Balaguer Vintró I, Bernades Bernat E. Factores de riesgo e incidencia de nuevos casos en el estudio prospectivo de la cardiopatía isquémica de Manresa. Rev Esp Cardiol 1976;29:127-35

13. Tomás Abadal L. Estudio prospectivo sobre la cardiopatía coronaria de Manresa:: 15 años de seguimiento. Rev Clin Esp 1887; 180: 4-7.

14. Tomás Abadal L, Varas Lorenzo C, Bernades Bernat E, Balaguer Vintró I. Coronary risk factors and 20-year incidence of coronary heart disease and mortality in a Mediterranean industrial population. The Manresa Study, Spain. Eur Heart J 1994; 15: 1028-36.

15. The ERICA Research Group. The CDH risk-map of Europe. The 1st report of the WHO-Erica project. Eur Heart J 1988: 9 suppl I: 1-36.

16. World Health Organization European Collaborative Group. Multifactorial Trial in the prevention of coronary heart disease: I. Recruitment and initial findings. Eur Heart J 1980; 1:72-80.

17. WHO Monica Project/Keil U and Kuulasma K. WHO MONICA Project: Risk Factors . Int J Epidemiol 1989;18: S46-S55

18. Actualidad Tabaquera Madrid: Servicio de Estudios de Tabacalera I; 1981.

19. Keys A, Menotti A, Aravanis Ch. The Seven Countries Study: 2.289 death in 15 years. Prev Med $1984 ; 13: 141-54$

20. Gey KF, Puska P, Jordan P, Moser UK. Inverse correlation between plasma vitamin $\mathrm{E}$ and mortality from ischaemic heart disease in cross cultural epidemiology. Am J Clin Nutr 1991; 53 supl: 326S$334 \mathrm{~S}$

21. Renaud S, De Lorgeril M. Wine, alcohol, platelets and the French paradox for coronary heart disease. Lancet 1992;339:1523-6.

22. Law M, Wald N. Why heart disease mortality is low in France: the time lag explanation. Br Med J 1999; 318: $1471-80$
23. Pekkanen J, Nissinen A, Puska P, Punsar S, Karvonen MJ. Risk factors and 25 year risk of coronary heart disease in male population with high incidence of the disease : the Finnish cohorts of the Seven Countries Studies BMJ 1989: 299:81-5.

24. Haffner SM, D’Agostino R Jr., Mykkanen L, Tracy R, Howard B, Rewers M, Selby J, Savage PJ, Saad MF. Insulin sensitivity in subjects with type 2 diabetes. Relationship to cardiovascular risk factors: The insulin resistance Atherosclerosis Study. Diabetes Care, 1999;22: 562-8.

25. Calles Escandon J, García Rubí E, Mirza S, Mortensen A. Type 2 diabetes: one disease, multiple cardiovascular risk factors. Coron Artery Dis 1999;10:23-30.

26. Stokes III J, Kannel WB, Wolf PA, The relative importance of selected risk factors for varius manifestations of cardiovascular disease among men and women from 35 to 64 years old: 30 years of follow-up in the Framingham Study. Circulation 1987; 75 : 56V-73V.

27. Colhoun HM, Dong W, Barakat MT, Mather HM, Poulter NR. The scope for cardiovascular disease risk factor intervention among people with diabetes mellitus in England: a population-based analysis from the Health Surveys for England 1991-94. Diabet Med 1999;16:35-40.

28. Orchard TJ, Stevens LK, Forrest KY, Fuller JH Cardiovascular disease in insulin dependent diabetes mellitus: similar rates but different risk factors in the US compared with Europe. Int J Epidemiol 1998; 27: 976-983.

29. López García Aranda V, González López M, Trujillo Berraquero F, Rojas Rodríguez J. Influencia de los factores de riesgo coronario en prevención secundaria: tabaco. Rev Esp Cardiol 1998; 51 supp 6: 10-7.

30. Mariotti S, Capocaccia R, Farchi G. Age, period, cohort and geographical area effects on the relationship between risk factors and coronary heart disease mortality. 15 year follow-up of the European cohorts of the Seven Countries Study. J Chron Dis 1986;39: 229-42. 